\title{
High compressibility caused by particle breakage: a DEM investigation
}

\author{
Marta Stasiak ${ }^{1}$, Vincent Richefeu ${ }^{1, *}$, Gaël Combe ${ }^{1, * *}$, Jad $_{\text {Zghondi }}{ }^{2}$, and Gilles Armand ${ }^{2}$ \\ ${ }^{1}$ Univ. Grenoble Alpes, CNRS, Grenoble INP, 3SR, 38000 Grenoble, France. \\ ${ }^{2}$ Andra, R\&D Division, Meuse/Haute-Marne Underground Research Laboratory, 55290 Bure, France.
}

\begin{abstract}
This paper summarises the numerical and experimental studies of brittle, hollow, cylindrical particles, called shells. It addresses the influence of shell properties both at the particle and assembly scales. Extreme compressibility has been recorded in the oedometer tests. Due to the large internal porosity of the shell, the breakage phenomena lead to highly compressive deformations with a significant stress dissipation. Using the Discrete Element Method (DEM), we have investigated in depth the micro-mechanical phenomena governing this macroscopic response. By quantifying the breakage and separating the double-porosity of the material, the foundations of a future constitutive model have been established throughout a simple prediction model applicable to the engineering practice.
\end{abstract}

\section{Introduction}

This article is dedicated to the numerical and experimental investigations of an assembly of brittle, hollow, cylindrical particles, called shells; Figure 1(a). Andra ${ }^{1}$ have incorporated this granular material into a pre-precasting technology of a mono-block double-layer tunnel lining. Shells create the core of the segment's outer layer, placed onto the inner concrete arch (US pending patent of Andra and consulting group CMC [1]). The shell-based layer experiences significant particle breakage under a sufficient load exerted by the converging host-rock. Due to the large internal porosity of the shell, the breakage phenomenon leads to highly compressive deformations with simultaneous stress dissipation. This work has investigated the origins of the high compressibility of the shell layer using an oedometer test (imposed uniaxial compression with otherwise geometrically constrained boundaries). The modelling relays on Discrete Element Method (DEM) particularly well suited for granular materials [2]. Then, some additional techniques offer a choice of a way to create a reliable breakage model. Both fragment substitution (FS) and particle bonding (BP) techniques have already received considerable attention. In FS, a native particle is removed and replaced by a set of new, smaller, self-similar offspring particles after the material failure [3-5]. In BP technique, the offspring particles are generated a priori and joined together to form the native particle through the cohesive, breakable contacts [6-8]. Hereinafter, the shell is generated as Bonded Particle (BP) made of longitudinal polygonal-like sectors; Figure 1(b).

Section 2 is dedicated to the shell-scale study and explains how the shell model was structured. Section 3

\footnotetext{
*e-mail: vincent.richefeu @3sr-grenoble.fr

**e-mail: gael.combe@3sr-grenoble.fr

${ }^{1}$ French acronym for the national agency for radioactive waste management.

A video is available at https://doi.org/10.48448/az20-zt49
}

is a brief remark on the process of the parameter selection. Section 4 focuses on shell packing. Comparison with a typical experimental behaviour verifies the modelling technique at the macro-scale. This part comments also on the limitations and complexity emerging from the model characteristics. Section 5 is an elaborate investigation of high compressibility bridging micro- and macromechanics. The analysis includes separation of double porosity and quantification of pore collapse. Both concepts are then used in structuring the foundation of a 1D constitutive model. Section 6 concludes on the article and announces the upcoming part.

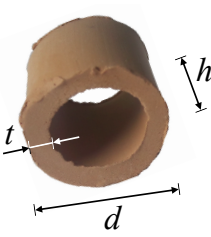

(a)

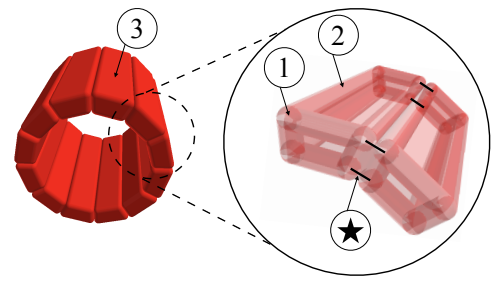

(b)
Figure 1. (a) Crushable shell made of backed clay. The tubular geometry is described by diameter $d$, thickness $t$ and height $h$ such that $d \approx h \approx 20 \mathrm{~mm}$ and $t \approx 2.5 \mathrm{~mm}$. (b) A cluster is a numerical representation of shell generated with 12 rigid sectors bonded by four corner-to-corner links $\circledast$. Each sector is build from the (1) - corner spheres, (2) - tubular edges and (3) - side plates.

\section{Generation of a breakable model}

In the current paper, a brittle particle having a tubular shape is referred to as shell; Figure 1(a). Its numerical representation was named cluster and is an aggregate of orderly joint rigid constituents, the sectors. Figure 1(b) shows that each sector is constructed from simpler 3D elements to achieve a sphero-polygonal shape. The links, 
marked by $\circledast$ in Figure 1(b), are distinctive cohesive interaction points between adjacent sectors. For the sake of simplicity, links are restricted to corner-to-corner interactions between sectors. Considering two directions separately, link forces $\left(f_{I}\right.$ and $\left.f_{I I}\right)$ are always calculated under an assumption of linear elasticity. The forces can develop up to yield thresholds:

- $f_{I}^{\star}$ - pure tensile force causing mode- $I$ crack opening in a normal direction. Its value has been easily deduced from the experimental campaign [9],

- $f_{I I}^{\star}$ - pure shear force causing in-plane mode- $I I$ fracture in a tangential direction.

Those thresholds not only define the rupture of the link in pure mode- $I$ or mode- $I I$ conditions but are also necessary to verify a mixed-mode. It is achieved through a parabolic failure envelope (Eq. 1) which involves a shape parameter, i.e., the power of second left side term, that can tune the reciprocal dependence of failure-modes. When a single link breaks, the remaining links belonging to the same predefined rupture-surface between sectors break as well. The sectors are then free to re-contact by classical linearelastic contacts with Coulombic friction. These contacts appear also in-between clusters within the packing.

This particular modelling choice requires 8 micromechanical inputs (two yield thresholds, four micro stiffnesses, a friction coefficient and a shape of failure envelope). For a deeper explanation of the link and contact laws, the interested reader can turn to [10].

$$
\frac{f_{I}}{f_{I}^{\star}}+\left(\frac{f_{I I}}{f_{I I}^{\star}}\right)^{2}=1 .
$$

\section{Parameter selection}

A great deal of thoughts is necessary before reaching a conclusion about a selection of the modelling parameters. In the field of discrete modelling there are many parameters of various character: (i) micro-mechanical inputs as introduced in Section 2, (ii) purely numerical parameters required by the algorithm, such as a time step or a frequency for rebuilding neighbourhood list, (iii) internal variables of the assembly (initial density, shell orientations, and so forth). A preliminary parameter choice has been made by bridging the shell-scale experiment and modelling [10]. No doubt our experimental endeavours to classify the variability of tensile strength, the angle of friction and a distribution shell orientation were substantial to the calibration process [11]. Still, to complete a suitable parameter selection, it was necessary to probe the sensitivity of the model to many parameters at the sample-scale in an extensive modelling campaign $[10,11]$. Eventually, among many parameters, the compressive behaviour was found wholly subservient to two of them: the initial density and the tensile strength of shell $\left(f_{I}^{\star}\right.$-controlled).

\section{Oedometer test}

After choosing the parameters, the natural step is to probe the cluster model in the case of a shell packing. Just like the actual loading in experiments, the sample has been compressed uni-axially with the boundaries fixed in the remaining directions. Such loading conditions are imposed in a test commonly recognised as the oedometer test. The experimental stress-controlled test has been conducted by Euro-Géomat-Consulting [12] on a larger cylindrical sample (diameter $D=30 \mathrm{~cm}$ and initial height $H_{0} \approx 13 \mathrm{~cm}$ ). A strain-imposed test was modelled with an in-house code RocKABLE (developed by V. Richefeu). The assembly comprised 1926 clusters (12 sectors each) and conformed to the physical sample size. Figure 2 illustrates a typical, highly compressive response (axial stress $\sigma_{a}$ vs. axial strain $\varepsilon_{a}$ ) recorded experimentally. Three zones can be distinguished: $(i)$ a "stress-plateau" in high-compressibility, (ii) a curvature of the trend suggesting that the density level is increasing and (iii) a strongly densified state (or in other words hard-densification), when the stress rises abruptly. Figure 2 shows also the simulated version of the curve. It is clear that the cluster model is capable of capturing the compressive response respecting those three distinctive zones. The good match of the numerical curve up to $\varepsilon_{a} \approx 45 \%$ confirms the appropriate choice of micro-mechanical parameters, but the geometry of the sector (both its shape and its size) inevitably restricts the compression ability. This is an inherent limitation of the cluster model: longitudinal (rigid) sectors are simply incapable of breaking into smaller fractions as was observed in the experiments. It is reasonable to think that, with a high number of simulations, such compromise on particle size should be acceptable to keep the computation times achievable. Nevertheless, the high compressibility phenomenon can still be reliably investigated, at least up to the bifurcation point (at $\varepsilon_{a} \approx 45 \%$ ).

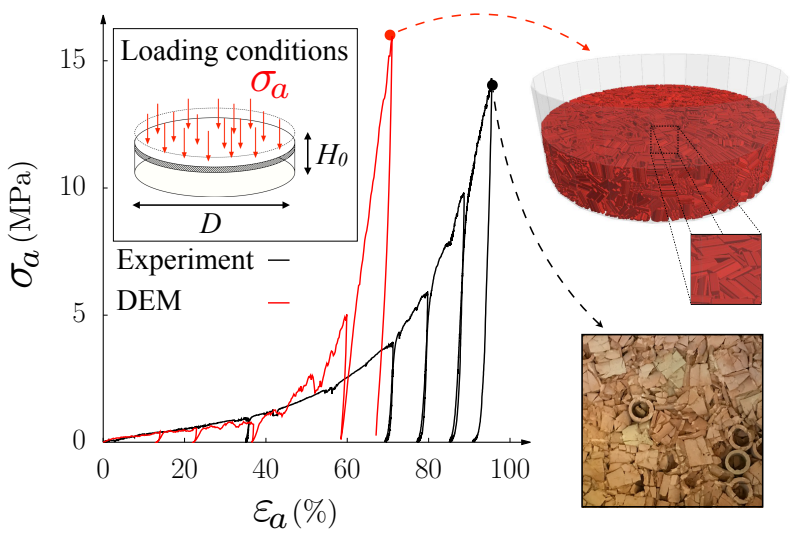

Figure 2. Comparison of the macroscopic mechanical behaviour to oedometeric compression. The experiment was performed at Euro-Géomat-Consulting on a sample with size: $D=30 \mathrm{~cm}$ and $H_{0} \approx 13 \mathrm{~cm}$ [12]. Equivalent DEM packing of 1926 clusters, that is 23112 sectors; Figure 1(b). Right-side insets present crushing at the final stage.

\section{High compressibility}

Prior to our numerical study, shell breakage has been witnessed in the experimental campaign. Understandably, it can be assumed that pore collapse, brought up by a primary shell breakage, is of an essential significance to the 
highly compressive regime. To better exploit the advantages of the compressible layer, the importance of shell internal porosity has to be more transparent. The current section is devoted to the pursuit of a deeper understanding. Even though the conclusions result from the numerical investigations, they are projected on reality.

A much simpler way of dealing with shell breakage is to assume that the primary breakage separates a shell axially in at least two large moon-shaped fragments. Further fragmentation is temporarily disregarded due to the highly porous tubular geometry of the shell. The breakage level $b$ can consequently be defined as a simple portion of how many clusters of the initial assembly $\left(N_{0}\right)$ were broken $\left(N_{\text {broken }}\right)$ :

$$
b=\frac{N_{\text {broken }}}{N_{0}} .
$$

By definition, $b$ ranges between 0 (no breakage) and 1 (all clusters were crushed).



Figure 3. Evolution of breakage ratio $b$ (red curve and leftside axis) in three distinctive zones (I - (III) of the mechanical behaviour (black curve and right-side axis).

In Figure 3, the rise of $b$, that is the growth of pore collapses (red curve), confronted with the compression resistance (black curve) clearly demonstrates a mutual relationship at the respective stages of evolution.

After initial shell rearrangements in regime (I) $(b<$ $0.05)$, the intensity of pore collapse is at its strongest - zone (II). The high compressibility with the "stressplateau", most interesting to us, is achieved exactly when numerous voids rapidly became accessible. With the inhibition of primary breakage in phase (II), the sample is progressively losing the extreme compressibility in order to, inevitably, experience the final hard-densification (for $b>0.95$ ). This observations confirm that, indeed, the secondary breakage is overshadowed by the pore collapse for this material.

The dilemma faced now is how to take advantage of the above observations? In the field of soil mechanics, void ratio $e$ is a well established dimensionless parameter measuring a volume of all the voids $\left(V_{\mathrm{v}}\right)$ over a volume of the solid skeleton $\left(V_{\mathrm{s}}\right)$ :

$$
e=V_{\mathrm{v}} / V_{\mathrm{s}}=\left(V_{\mathrm{tot}}-V_{\mathrm{s}}\right) / V_{\mathrm{s}},
$$

where $V_{\text {tot }}$ is a total volume of a sample.

Figure 4 shows that $e$ can only decrease (black curve), here with a trend specific for strain-controlled uniaxial compression. Now, a redefinition of what is pore and what is solid might be reconsidered according to volumes that are accessible $\left(V_{\text {inaccessible }}\right)$ or inaccessible $\left(V_{\text {inaccessible }}\right)$; Eq. (4). As long as a cluster remains intact, the volume of its internal hollow $\left(V_{\mathrm{v}}^{i}\right)$ is hardly accessible to other particles, and thus treated as a part of the solid phase. When the cluster breaks, its internal void becomes reachable. These features suggest the following modified definition of the void ratio that accounts for the rate $b$ of broken clusters:

$$
e^{\star}=V_{\text {accessible }} / V_{\text {inaccessible }}=\left(V_{\mathrm{v}}-V^{\mathrm{o}}\right) /\left(V_{\mathrm{s}}+V^{\mathrm{o}}\right),
$$

where

$$
V^{\mathrm{o}}=(1-b) \sum_{i=0}^{N_{0}} V_{\mathrm{v}}^{i}
$$

This new idea causes a peak to appear in the evolution of $e^{\star}$ as shown in Figure 4. The curve rises as long as the intense breakage lasts, best pronounced in phase (II). The peak is reached once $b \approx 0.95$, that is when the major part of internal porosity has been already exploited, and finally, the hard-densification launches the high stress. When all clusters are crushed, $b=1, e^{\star}$ becomes $e$ by definition, and therefore, the curves must merge. Figure 4 makes the great value of this concept clear, as $e^{\star}$ actually highlights the highly compressible regime of the response. By tuning the peak of $e^{\star}-$ this can be done by changing the shell thicknesses for example - the compressibility can be ruled. This would provide a tool to optimise the material strength

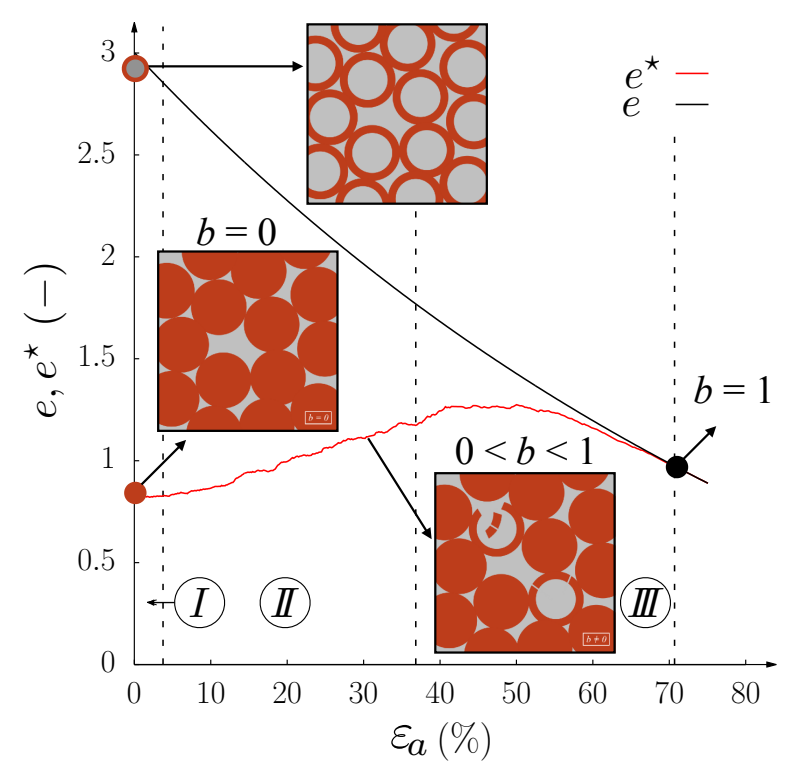

Figure 4. Void ratio evolution; Eq. (3) and modified void ratio $e^{\star}$; Eq. (4) based on breakage level with respect to vertical strains (large strain definition). 
and the geometrical parameters. A prediction of the $e^{\star}-$ $\varepsilon_{a}$ relationship is however just a first step to design more functional constitutive model for materials with extreme internal porosity $\left(e^{\star}-\sigma_{a}\right)$.

In the current paper, it is only possible to present a short essence of full development. The following mathematical expression was developed from some simple geometrical definitions:

$$
e^{\star}\left(\varepsilon_{a}\right)=\frac{e\left(\varepsilon_{a}\right)-\left(V^{\mathrm{o}} / V_{\mathrm{s}}\right)}{1+\left(V^{\mathrm{o}} / V_{\mathrm{s}}\right)},
$$

where $e\left(\varepsilon_{a}\right)$ is the conventional void ratio with an initial value of $e_{0}$ (input) and its evolution is imposed by the strain-control; Figure 4. The breakage level $b$ is introduced in the model through $V^{\mathrm{o}}$, recall Eq. (5), by assuming a linear evolution, as in Figure 3 in phase (II).

Figure 5 presents the outcome of the prediction model for different thicknesses $t$ of the cluster. These predictions are also compared with the corresponding simulations (dashed lines). The prediction works only on the average point of view and does not reproduce subtleties such as a short post-peak decrease of $e^{\star}$ before the curves merge. It is still remarkable to observe that, although built upon very basic developments and assumptions, the prediction model captures quite well the main features of $e^{\star}$ evolution. Essentially, this geometrical model established very solid foundations for more complex perdition of the constitutive behaviour that, sadly, is left to be discussed elsewhere.

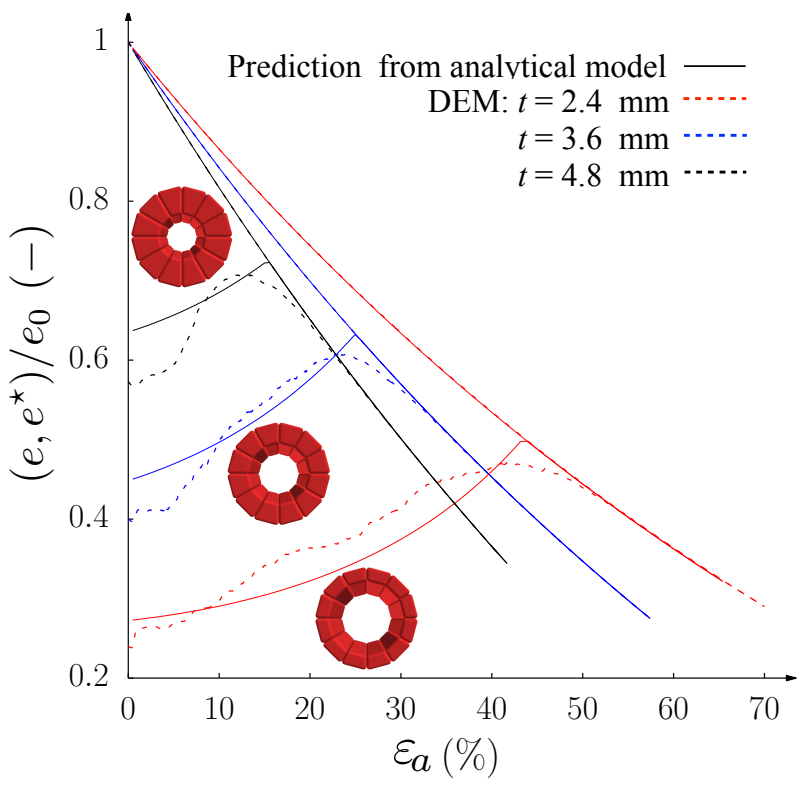

Figure 5. Normalised evolution of void ratio $e / e_{0}$ and its redefinition $e^{\star} / e_{0}$. DEM curves (dashed lines) and predictions (solid lines), Eq. (6), for clusters with various pore sizes, achieved by through shell thickness $t$ with constant external diameter $d$. Linearity of breakage level was adjusted as follows: $b=$ $0.024 \varepsilon_{a}$ (red), $b=0.04 \varepsilon_{a}$ (blue), and $b=0.065 \varepsilon_{a}$ (black).

\section{Conclusion}

Hereinbefore, we have presented a discrete modelling technique with particle clustering: both in non-rigid (to simulate the particle breakage) and rigid (to construct more complex shape) manners. The model structured the tubular cluster (our crushable particle) as a set of 12 sectors joined by cohesive links. The links imitated the material cohesion by two yield thresholds (tensile and shear), however, in oedometer test the importance of tensile threshold was more pronounced. It has been demonstrated that such a model, if suitably calibrated, archives most satisfactory results when compared with experimental behaviour. Furthermore, thanks to discrete modelling it was possible to investigate the effect of micromechanical breakage on the highly compressible macroscopic response observed experimentally. Cluster breakage not only dissipates the macroscopic stress but also releases access to the large internal pores enabling large compressive deformations. We used this insight to redefine the void ratio using pore accessibility. Essentially, we have observed that the inter-particle void is increasing when the breakage is intense, whereas it would only decrease if the separation of internal and inter-particle porosity is missing. To take the advantage of high compressibility one must aim the incline in primary breakage be sharp as long as possible. We have, therefore, constructed a foundation of a future constitutive model, that despite its simplicity already could be useful to predict the highly compressible regime of material.

The Laboratoire 3SR is part of the LabEx Tec 21 (Investissements dâAvenir, Grant Agreement No. ANR-11-LABX0030).

\section{References}

[1] Andra, CMC, Patent, FR3021346 (2016)

[2] P.A. Cundall, O.D.L. Strack, Géotechnique 29, 47 (1979)

[3] O. Tsoungui, D. Vallet, J.C. Charmet, Powder Technol. 105, 190 (1999)

[4] O. Ben-Nun, I. Einav, Philos. Trans. R. Soc. A 368, 231 (2010)

[5] J.P. de Bono, G.R. Mcdowell, Granul. Matter 18 (2016)

[6] C. Thornton, K.K. Yin, M.J. Adams, J. Phys. 29, 424 (1996)

[7] M.D. Bolton, Y. Nakata, Y.P. Cheng, Géotechnique 58, $471(2008)$

[8] J.F. Wang, H.B. Yan, Procedia Eng. 14, 1713 (2011)

[9] M. Stasiak, G. Combe, J. Desrues, V. Richefeu, P. Villard, G. Armand, J. Zghondi, EPJ Web Conf. 140, 07015 (2017)

[10] M. Stasiak, G. Combe, J. Desrues, V. Richefeu, P. Villard, G. Armand, J. Zghondi, in Micro to MACRO Mathematical Modelling in Soil Mechanics. Trends in Mathematics (Birkhäuser, Cham, 2018)

[11] M. Stasiak, Uniaxial compression of a highly crushable granular material - a 3D DEM study (Université Grenoble Alpes, 2019)

[12] B.Q.H. Ly, J.C. Robinet, Tech. Rep. CRP0EUG170004, Euro-Géomat-Consulting (2017) 